\title{
ELLIPTIC AND PARABOLIC BMO AND HARNACK'S INEQUALITY
}

\author{
HUGO AIMAR
}

\begin{abstract}
We give a generalization of the John-Nirenberg lemma which can be applied to prove $A_{2}$ type conditions for small powers of positive solutions of elliptic and parabolic, degenerate and nondegenerate operators.
\end{abstract}

One of the main tools in the original Moser proof of Harnack inequalities for elliptic and parabolic equations is a John-Nirenberg type lemma concerning the behavior of the distribution of a function satisfying a bounded mean oscillation property $\left[\mathbf{M}_{1}, \mathbf{J N}\right.$ and $\left.\left.\mathbf{M}_{2}\right]\right)$. This technique has extensively been used in different elliptic situations. For example: in [FKS], Fabes, Kenig and Serapioni use the John-Nirenberg lemma for BMO $(w d x)\left(w \in A_{\infty}\right)$ proved by Muckenhoupt and Wheeden in [MW], in order to prove regularity of solutions of degenerate elliptic operators. In [FL and FS], Franchi, Lanconelli and Serapioni apply the JohnNirenberg type inequality proved by Burger in [Bu] on spaces of homogeneous type, in order to extend Moser's technique to different degenerate elliptic operators. In the parabolic case the situation is different since Moser $\left[\mathbf{M}_{3}\right]$ has obtained another proof of Harnack's inequality by an extension of Bombieri's argument [B], which does not use John-Nirenberg type lemmas. The degenerate parabolic situation studied by Chiarenza and Serapioni $\left[\mathbf{C S}_{1}\right.$ and $\left.\mathbf{C S}_{2}\right]$ follows the pattern of $\left[\mathbf{M}_{3}\right]$.

In a recent paper, $[\mathbf{F G}]$, Fabes and Garofalo returned to the original Moser proof of parabolic Harnack inequality. The main point is their extension of A. P. Calderón's proof of the John-Nirenberg lemma [N]. In their subdivision and selection process, the rectangular geometry of the parabolic balls and the translation nature of the time lag play an important role.

On the other hand it is well known that the usual (elliptic) selection process can be carried over to the general setting of spaces of homogeneous type applying Wiener type covering lemmas. See, for instance, Macías and Segovia [MS].

The main purpose of this note is to give an extension of the John-Nirenberg lemma to the setting of spaces of homogeneous type with general "time lags", in such a way that both, degenerate and nondegenerate, elliptic and parabolic situations, become particular cases.

In $\S 1$ we state the result, and its applications and examples. In $\S 2$ a JohnNirenberg type lemma is proved. In $\S 3$ we shall prove Theorem (1.7).

1. Statement of the result. Applications. Let $X$ be a set, a nonnegative symmetric function $d$ on $X \times X$ shall be called a quasi-distance if there exists a

Received by the editors July 8, 1986.

1980 Mathematics Subject Classification (1985 Revision). Primary 42B99; Secondary 35J15, $35 \mathrm{~K} 10$. 
constant $K_{0}$ such that $d(x, y) \leq K_{0}(d(x, z)+d(z, y))$ for every $x, y, z \in X$, and $d(x, y)=0$ if and only if $x=y$. Let $\mu$ be a positive measure defined on a $\sigma$-algebra of subsets of $X$ which contains the $d$-balls and satisfies the following inequalities $0<\mu(B(x, 2 r))<A \mu(B(x, r))<\infty$, for some constant $A$, every $x \in X$ and $r>0$. We shall say that $(X, d, \mu)$ is a space of homogeneous type if $X$ is a set endowed with a quasi-distance and a measure satisfying these conditions. In this note we shall consider a space of homogenous type for which continuous functions are dense in $L^{1}$. If $f$ is a positive measurable function on $X$ and $E$ is a measurable subset of $X, m_{E}(f)$ means $\mu(E)^{-1} \int_{E} f d \mu$.

(1.1) DEFinition. Let $B\left(x_{0}, R_{0}\right)$ be a fixed ball in the space of homogeneous type $(X, d, \mu)$. We shall say that a function

$$
T: B\left(x_{0}, R_{0}\right) \times\left(0, R_{0}\right] \rightarrow X \times \mathbf{R}^{+} ; \quad T(x, r)=(\xi, \rho),
$$

is a lag mapping if there exist three constants $K_{i}, i=1,2,3$, such that the inequalities

$$
\begin{gathered}
d(x, \xi) \leq K_{1} r, \\
K_{2} \rho \leq r \leq K_{3} \rho,
\end{gathered}
$$

hold for every $x \in B\left(x_{0}, R_{0}\right)$ and $r \in\left(0, R_{0}\right]$.

In the definition of BMO we shall use nonnegative functions $h$ of the real variable $t$, which share the following properties with small powers of $t^{+}=(t+|t|) / 2: h$ is continuous, nonnegative, $h(t)=0$ if $t<0, h(t)$ is increasing for $t>0, h(t+s) \leq$ $h(t)+h(s)$ and $e^{-\varepsilon h(t)}$ is an integrable function on $(0, \infty)$ for every $\varepsilon>0$.

(1.4) DEFINITION. Let $f$ be a real-valued measurable function on the space of homogeneous type $(X, d, \mu)$. We shall say that $f$ satisfies a BMO condition with lag mapping $T$ on the ball $B\left(x_{0}, R_{0}\right)$, with respect to $h$, if there exists a real function $C(x, r)$ on $B\left(x_{0}, R_{0}\right) \times\left(0, R_{0}\right]$ such that

$$
\begin{aligned}
& m_{B(x, r)}(h(f-C(x, r))) \leq N(f), \\
& m_{B(\xi, \rho)}(h(C(x, r)-f)) \leq N(f),
\end{aligned}
$$

for some constant $N(f)$ and every $(x, r) \in B\left(x_{0}, R_{0}\right) \times\left(0, R_{0}\right]$. Let $\operatorname{BMO}\left(x_{0}, R_{0}, T, h\right)$ be the class of all such functions.

The main result of this note is the following.

(1.7) THEOREM. Let $T$ be a one-to-one lag mapping on $B\left(x_{0}, R_{0}\right)$ with the following property: there exists $0<\gamma<1$ such that for every $r \leq R_{0}$

$$
B\left(x_{0}, \gamma r\right) \times(0, \gamma r] \subset T\left(B\left(x_{0}, r\right) \times(0, r]\right) .
$$

Then there exist two constants $\delta$ and $C$ depending only on $K_{i}, A, \gamma$, and a lag mapping $S$ on $B\left(x_{0}, \delta R_{0}\right)(S(x, r)=(\zeta, \tau))$ such that if $f \in \operatorname{BMO}\left(x_{0}, R_{0}, T, h\right)$ and $u=e^{-f}$, the inequality

$$
\left(m_{B(x, r)}\left(u^{-\varepsilon}\right)\right) \cdot\left(m_{B(\varsigma, \tau)}\left(u^{\varepsilon}\right)\right) \leq C
$$

holds for some $\varepsilon>0$, depending only on $N(f)$, and every $(x, r) \in B\left(x_{0}, \delta R_{0}\right) \times$ $\left(0, \delta R_{0}\right]$. Moreover, the mapping $S$ can be given explicitly in terms of $T$ :

$$
S(x, r)=(\xi(x, r / \eta) ; \eta \rho(x, r / \eta))
$$


where $\xi$ and $\rho$ are the components of $T$ and $\eta$ is a constant depending only on $K_{i}$, $i=0,1,2,3$.

Roughly speaking, Theorem (1.7) asserts that if $f$ is of bounded mean oscillation with lag mapping $T$, then some positive power of $e^{-f}$ belongs to an $A_{2}$ class with a lag mapping closely related to $T$.

In the next paragraph, examples and applications are given.

(1.10) Elliptic case: $\left[\mathbf{M}_{1}\right.$ and $\left.\mathbf{J N}\right]$ Let

$$
L u=\sum_{i, j=1}^{n} \frac{\partial}{\partial x_{i}}\left(a_{i j} \frac{\partial u}{\partial x_{j}}\right),
$$

where $a_{i j}(x)$ is a measurable symmetric matrix satisfying the inequalities $\lambda|\xi|^{2} \leq$ $a_{i j}(x) \xi_{i} \xi_{j} \leq \Lambda|\xi|^{2}, \lambda$ and $\Lambda$ constants. If $u$ is a positive solution of $L u=0$ on the cube $Q_{0}$, using Poincaré's inequality, Moser proves the usual BMO property for $v=\log 1 / u$ on the cube $2^{-1} Q_{0}$ :

there exist a constant $C=C(n, \lambda, \Lambda)$ and, for each subcube $Q$ of $2^{-1} Q_{0}$, a number $C(Q)$ such that

$$
\frac{1}{|Q|} \int_{Q}|v-C(Q)| \leq C .
$$

This condition is clearly equivalent to (1.5) and (1.6) with $h(t)=t^{+}$and $T$ the identity. Then, Theorem (1.7) gives the usual $A_{2}$ condition for $u^{\varepsilon}$, since $S$ is also the identity.

(1.11) Degenerate elliptic case I: [FKS and MW]. The coefficients $a_{i j}$ of $L$ satisfy the weaker condition $\lambda w(x)|\xi|^{2} \leq a_{i j}(x) \xi_{i} \xi_{j} \leq \Lambda w(x)|\xi|^{2}$ with $w \in A_{2}$. The weighted extension of Poincaré's inequality gives a weighted BMO for $v$. Theorem (1.7) applies to the situation $X=\mathbf{R}^{n}, d \mu=w d x, d$ the euclidean distance in $\mathbf{R}^{n}$ and $T$ the identity.

(1.12) Degenerate elliptic case II: [FL, FS and Bu]. The condition on $a_{i j}$ is of the form $\lambda w(x) \lambda_{j}^{2}(x) \xi_{j}^{2} \leq a_{i j}(x) \xi_{i} \xi_{j} \leq \Lambda w(x) \lambda_{j}^{2}(x) \xi_{j}^{2}$, where $w \in A_{2}$ on the space of homogeneous type with distance given by the vector $\left(\lambda_{1}, \lambda_{2}, \ldots, \lambda_{n}\right)$ (see [FS]). Theorem (1.7) applies again to obtain a weighted $A_{2}$ condition on balls in such a distance.

(1.13) Parabolic case: $\left[\mathbf{M}_{2}, \mathbf{M}_{3}\right.$ and $\left.\mathbf{F G}\right]$. Let $u$ be a positive solution of $\partial u / \partial t=$ $L u, L$ as in (1.10). Poincaré's inequality gives now a "parabolic BMO with time lag": for every parabolic subrectangle $R$ of the unit cube in $\mathbf{R}^{n+1}$ there exists $C(R)$ such that

$$
\frac{1}{\left|R^{+}\right|} \int_{R^{+}} \sqrt{(v-c(R))^{+}} d x d t \leq C
$$

and

$$
\frac{1}{\left|R^{-}\right|} \int_{R^{-}} \sqrt{(c(R)-v)^{+}} d x d t \leq C
$$

where $R^{+}$and $R^{-}$are the upper and lower halves of $R$. The structure of space of homogeneous type is $\left(\mathbf{R}^{n+1}, d, \lambda\right)$, where $\lambda$ is Lebesgue measure and $d$ is the translation invariant distance defined by

$$
d((x, t) ;(0,0))=\max \{|x|, \sqrt{|t|}\} ; \quad x \in \mathbf{R}^{n}, t \in \mathbf{R} .
$$


The lag mapping $T$ is given by

$$
T((x, t) ; r)=\left(\left(x, t-2 r^{2}\right) ; r\right) .
$$

It is easy to see that $(1.8)$ is satisfied. Since $S((x, t) ; r)=\left(\left(x, t-2 r^{2} / \eta^{2}\right) ; r\right)$ and $\eta<1$, then we have the expected lag proportional to $r^{2}$ in the $A_{2}$ condition for a positive power of $u$, which takes the form

$$
m_{R_{1}}\left(u^{-\varepsilon}\right) \cdot m_{R_{2}}\left(u^{\varepsilon}\right) \leq C
$$

needed to apply Moser's method.

(1.14) Degenerate parabolic case. As we said in the Introduction, a class of degenerate parabolic operators has been studied by Chiarenza and Serapioni $\left[\mathbf{C S}_{1}\right.$ and $\mathbf{C S}_{2}$ ]. There, Harnack's inequality is obtained when the degeneration of the elliptic part is as in (1.11) with the weight $w$ belonging to Muckenhoupt's class $A_{1+2 / n}$. The method used in $\left[\mathbf{C S}_{1}\right.$ and $\left.\mathbf{C S}_{2}\right]$ is that of Bombieri and Moser and consequently avoids John-Nirenberg type lemmas. The purpose of this paragraph is to prove that Theorem (1.7) applies in this situation.

Let us start by introducing a structure of space of homogeneous type on $\mathbf{R}^{n+1}$ associated to the $\mathbf{R}^{n}$ weight which defines the degenerate elliptic operator $L$. Let $w \in A_{p}\left(\mathbf{R}^{n}\right)$,

$$
A_{p}:\left(\int_{Q(x, r)} w\right)\left(\int_{Q(x, r)} w^{-1 /(p-1)}\right)^{p-1} \cong|Q(x, r)|^{p} .
$$

Let $h_{x}(r)=\left(\int_{Q(x, r)} w^{-1 /(p-1)}\right)^{p-1}$ and $h_{x}^{-1}$ be its inverse function. Let us define

$$
\begin{gathered}
d: \mathbf{R}^{n+1} \times \mathbf{R}^{n+1} \rightarrow \mathbf{R}^{+} \cup\{0\}, \\
d((x, t) ;(y, s))=|x-y|+h_{x}^{-1}(|t-s|)+h_{y}^{-1}(|t-s|)
\end{gathered}
$$

and $\mu=w \times \lambda$ on the Borel subsets of $\mathbf{R}^{n+1}$, here $\lambda$ is the one-dimensional Lebesgue measure and $w(E)=\int_{E} w d x$. Condition $A_{p}$ can be rewritten as

$$
A_{p}: \mu(B((x, t) ; r)) \cong r^{n p}
$$

where $B((x, t) ; r)$ is the $d$-ball with center $(x, t) \in \mathbf{R}^{n+1}$ and radius $r>0$. From this expression for $A_{p}$, the doubling property of $\mu$ is clear. The quasi-triangle inequality for $d$ follows from the fact that $w \in A_{\infty}$. Then $\left(\mathbf{R}^{n+1}, d, \mu\right)$ is a space of homogeneous type which fits the degenerate parabolic operator for $p=1+2 / n$. The family of balls in $\left(\mathbf{R}^{n+1}, d, \mu\right)$ is equivalent to the family of all rectangles of the form

$$
\tilde{B}((x, t) ; r)=Q_{(x, r)} \times\left(t-\frac{1}{2} h_{x}(r) ; t+\frac{1}{2} h_{x}(r)\right) .
$$

Let $u$ be a positive solution of

$$
\frac{\partial u}{\partial t}=\sum_{i, j=1}^{n} \frac{\partial}{\partial x_{i}}\left(a_{i j} \frac{\partial}{\partial x_{j}}\right) u
$$

on a cube of $\mathbf{R}^{n+1}$, where $A=\left(a_{i j}(x)\right)$ is a symmetric matrix of measurable functions satisfying the structural condition studied by Chiarenza and Serapioni, namely

$$
\lambda w(x)|\xi|^{2} \leq \sum_{i, j=1}^{n} a_{i j}(x, t) \xi_{i} \xi_{j} \leq \Lambda w(x)|\xi|^{2},
$$


where $w \in A_{1+2 / n}\left(\mathbf{R}^{n}\right)$. To apply Moser's method [ $\mathbf{M}_{2}$, p. 118] in order to obtain a BMO type condition for $v=\log 1 / u$, we need the following slight modification of the weighted Poincaré's inequality of Fabes, Kenig and Serapioni [FKS]:

(1.16) LEMMA. Let $0 \leq \varphi \leq 1$ be a continuous function with support in the cube $Q_{R}$ such that its level sets are convex sets. Then there is a positive constant $C$ such that the inequality

$$
\int|v-V|^{2} \varphi w \leq C R^{2} \int|\nabla v|^{2} \varphi w
$$

holds for every Lipschitz function, $v$, defined on $\overline{Q_{R}}$, where $V=\int v \varphi / \int \varphi$.

The function $v=\log 1 / u$ satisfies the following inequality

$$
\left.\int \psi^{2} v d x\right|_{t_{1}} ^{t_{2}}+\frac{1}{2} \int_{t_{1}}^{t_{2}} \int \psi^{2} \nabla v \cdot A \nabla v d x d t \leq 2 \int_{t_{1}}^{t_{2}} \int \nabla \psi \cdot A \nabla \psi d x d t
$$

where

$$
\psi=\psi(x)=\prod_{v=1}^{n} \chi_{v}\left(x_{v}\right)
$$

and

$$
\chi_{v}\left(x_{v}\right)=\left\{\begin{array}{l}
1 \text { if }\left|x_{v}-x_{v}^{0}\right|<r \\
0 \text { if }\left|x_{v}-x_{v}^{0}\right| \geq 2 r \\
\text { linear interpolation otherwise }
\end{array}\right.
$$

Therefore, from (1.11), we have

$$
\left.\int \psi^{2} v d x\right|_{t_{1}} ^{t_{2}}+\frac{\lambda}{2} \int_{t_{1}}^{t_{2}} \int|\nabla v|^{2} w \psi^{2} d x d t \leq 2 \Lambda\left(t_{2}-t_{1}\right) \int w|\nabla \psi|^{2} d x .
$$

Applying Lemma (1.16) and taking $t_{1} \rightarrow t_{2}$ we find that

$$
\frac{d V}{d t}+\frac{c}{\left|Q\left(x_{0}, r\right)\right| r^{2}} \int_{Q\left(x_{0}, r\right)}|v(x, t)-V(t)|^{2} w(x) d x \leq \frac{c^{\prime} w\left(Q\left(x_{0}, r\right)\right)}{r^{2}\left|Q\left(x_{0}, r\right)\right|} .
$$

Let $t_{0} \in \mathbf{R}, x_{0} \in \mathbf{R}^{n}$ and $0<r<1$ be fixed; $t_{1}=t_{0}-\frac{1}{2} h_{x_{0}}(r), t_{2}=t_{0}+\frac{1}{2} h_{x_{0}}(r)$ and $t \in\left(t_{1}, t_{2}\right)$. From (1.17) it is clear that

$$
\frac{d V}{d t} \leq c^{\prime} \frac{w\left(Q\left(x_{0}, r\right)\right)}{r^{2}\left|Q\left(x_{0}, r\right)\right|}=: m .
$$

Therefore $V-V_{1}:=V(t)-V\left(t_{1}\right) \leq m\left(t-t_{1}\right) \leq m h_{x_{0}}(r)$. For $s>3 m h_{x_{0}}(r)$ we set

$$
Q_{s}(t)=\left\{x \in Q\left(x_{0}, r\right): v(x, t)-V_{1}>s\right\} .
$$

If $x \in Q_{s}(t)$, then $v(x, t)-V>s+V_{1}-V>s-m h_{x_{0}}(r)>0$, consequently, from (1.17) we get

$$
\frac{d V}{d t}+c \frac{\left(s-V-V_{1}\right)^{2}}{\left|Q\left(x_{0}, r\right)\right| r^{2}} w\left(Q_{s}(t)\right) \leq m
$$

Integrating the above inequality from $t_{1}$ to $t_{2}$ and using our definition of $\mu$, we have

$$
\mu\left\{(x, t) \in \tilde{B}\left(\left(x_{0}, t_{0}\right) ; r\right): v(x, t)-V_{1}>s\right\} \leq c \frac{\left|Q\left(x_{0}, r\right)\right| r^{2}}{s-m h_{x_{0}}(r)}
$$


for every $s>3 m h_{x_{0}}(r)$. Therefore

$$
\begin{aligned}
& \int_{\tilde{B}\left(\left(x_{0}, t_{0}\right) ; r\right)} \sqrt{\left(v(x, t)-V_{1}\right)^{+}} d \mu(x, t) \\
& \quad \leq\left(3 m h_{x_{0}}(r)\right)^{1 / 2} \mu\left(\tilde{B}\left(\left(x_{0}, t_{0}\right) ; r\right)\right)+c \frac{\left|Q\left(x_{0}, r\right)\right| r^{2}}{\left(m h_{x_{0}}(r)\right)^{1 / 2}} .
\end{aligned}
$$

Since $w \in A_{1+2 / n}$, the first term on the right side is bounded by $c \mu\left(\tilde{B}\left(\left(x_{0}, t_{0}\right) ; r\right)\right)$, that the same bound is also true for the second term follows from Hölder's inequality. Now replacing $t$ by $2 t_{1}-t$ in the above argument, we get

$$
\begin{aligned}
& \int_{\tilde{B}\left(\left(x_{0}, t_{0}-h_{x_{0}}(r)\right) ; r\right)} \sqrt{\left(V_{1}-v(x, t)\right)^{+}} d \mu(x, t) \\
& \quad \leq c \mu\left(\tilde{B}\left(\left(x_{0}, t_{0}-h_{x_{0}}(r)\right) ; r\right)\right) .
\end{aligned}
$$

The preceding inequalities show that $v$ belongs to BMO with $h(s)=\sqrt{s^{+}}$and lag mapping satisfying (1.8), given by

$$
T((x, t) ; r)=\left(\left(x, t-h_{x}(r)\right) ; r\right) .
$$

Theorem (1.7) applies to show that the solution $u$ satisfies the following inequality

$$
\left(\int_{B((x, t) ; r)} u^{-\varepsilon} d \mu\right) \cdot\left(\int_{B((x, \tau) ; r)} u^{\varepsilon} d \mu\right) \leq c \mu(B((x, t) ; r))^{2}
$$

where $\tau=t-h_{x}(r / \eta)$.

2. A John-Nirenberg type lemma. The following Wiener type covering lemma can be found in $[\mathbf{C W}]$ with a slightly different statement.

(2.1) COVERING Lemma. Let $(X, d, \mu)$ be a space of homogeneous type. Let $B=\left\{B_{\alpha}=B\left(x_{\alpha}, r_{\alpha}\right): \alpha \in \Gamma\right\}$ be a family of balls in $X$ such that $\bigcup_{\alpha \in \Gamma} B_{\alpha}$ is bounded. Then, there exists a sequence of disjoint balls $\left\{B_{i}\right\} \subset B$ such that for every $\alpha \in \Gamma$ there exists $i$ satisfying $r_{\alpha} \leq 2 r_{i}$ and $B_{\alpha} \subset B\left(x_{i}, 5 K_{0}^{2} r_{i}\right)$.

In this section $B\left(x_{1}, R_{1}\right)$ is a fixed ball in the space of homogeneous type $(X, d, \mu)$, $T$ is a fixed lag mapping on $B\left(x_{1}, R_{1}\right)$ and $f$ is a fixed function in $\operatorname{BMO}\left(x_{1}, R_{1}, T, h\right)$ such that $N(f)=1$ and $C\left(x_{1}, R_{1}\right)=0$. In order to further simplify notation, we set

$$
M=K_{0}^{5}\left[2\left(K_{2}^{-1}+2 K_{1}\right) K_{3} K_{2}^{-1}+5 K_{0}^{2} K_{2}^{-1}+K_{1}\right]+1
$$

and

$$
\eta=\left[K_{0}\left(1+2 M K_{0}\right)\right]^{-1} \leq\left[K_{0}\left(1+2 K_{0}\right)\right]^{-1} .
$$

(2.2) Observation. For every $x \in B\left(x_{1}, \eta R_{1}\right)$ and every

$$
r \in\left[2 \eta K_{0} R_{1},\left(1 / K_{0}-\eta\right) R_{1}\right]=: I
$$

we have

$$
B\left(x_{1}, \eta R_{1}\right) \subset B(x, r) \subset B\left(x_{1}, R_{1}\right) .
$$

Since $\eta \leq\left[K_{0}\left(2 K_{0}+1\right)\right]^{-1}, I$ is nonempty. 
Let us consider the following modifications of $T(x, r)$ and $C(x, r)$ :

$$
\begin{aligned}
S(x, r) & =T(x, r) \quad \text { on }\left[B\left(x_{1}, R_{1}\right) \times\left(0, R_{1}\right)\right]-\left[B\left(x_{1}, \eta R_{1}\right) \times I\right], \\
& =T\left(x_{1}, R_{1}\right) \quad \text { on } B\left(x_{1}, \eta R_{1}\right) \times I, \\
\beta(x, r) & =C(x, r) \quad \text { on }\left[B\left(x_{1}, R_{1}\right) \times\left(0, R_{1}\right)\right]-\left[B\left(x_{1}, \eta R_{1}\right) \times I\right], \\
& =0 \text { on } B\left(x_{1}, \eta R_{1}\right) \times I .
\end{aligned}
$$

It is easy to see that $S$ is a lag mapping with constants depending on $\eta$. Moreover, if $S=(\sigma, \tau)$ we have

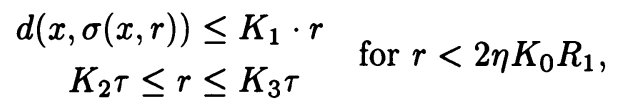

$$
\begin{array}{ll}
m_{B(x, r)}(h(f-\beta(x, r))) \leq 1 & \text { if } r<2 \eta K_{0} R_{1}, \\
m_{B(\sigma, \tau)}(h(\beta(x, r)-f)) \leq 1 & \text { everywhere. }
\end{array}
$$

(2.5) LEMMA. $f \in \operatorname{BMO}\left(x_{1}, R_{1}, S, h\right)$ with norm bounded by $A \eta^{-\log _{2} A}$.

ProOF. We only need to consider the case $(x, r) \in B\left(x_{1}, \eta R_{1}\right) \times I$. From Observation (2.2) we get

$$
\begin{aligned}
m_{B(x, r)}(h(f-\beta(x, r))) & =m_{B(x, r)}\left(h\left(f-C\left(x_{1}, R_{1}\right)\right)\right) \\
& \leq \frac{\mu\left(B\left(x_{1}, R_{1}\right)\right)}{\mu(B(x, r))} \cdot m_{B\left(x_{1}, R_{1}\right)}\left(h\left(f-C\left(x_{1}, R_{1}\right)\right)\right) \\
& \leq \frac{\mu\left(B\left(x_{1}, R_{1}\right)\right)}{\mu\left(B\left(x_{1}, \eta R_{1}\right)\right)} \leq A \eta^{-\log _{2} A} .
\end{aligned}
$$

For a given $\lambda>0$ set $\Omega^{\lambda}:=\left\{x \in B\left(x_{1}, \eta R_{1}\right): \beta(x, r)>\lambda\right.$ for some $r \in$ $\left.\left(0,\left(1 / K_{0}-\eta\right) R_{1}\right]\right\}$ and, for $x \in \Omega^{\lambda}$,

$$
R^{\lambda}(x):=\left\{r \in\left(0,\left(1 / K_{0}-\eta\right) R_{1}\right]: \beta(x, r)>\lambda\right\} .
$$

(2.6) LEMMA. Let $\lambda_{n}>\lambda_{n-1}>\cdots>\lambda_{1}>0$ and $\Omega^{k}=\Omega^{\lambda_{k}}$, then

(2.7) for every $x \in \Omega^{k}$ there exists $r^{k}(x)<2 \eta K_{0} R_{1}$ such that

$$
\beta\left(x, r^{k}(x)\right)>\lambda_{k} \geq \beta\left(x, M r^{k}(x)\right),
$$

(2.8) if $y \in \Omega^{k+1} \subset \Omega^{k}$ then $r^{k+1}(y) \leq r^{k}(y)$.

PROOF. Let $x \in \Omega^{n}$, then $R^{n}(x)=R^{\lambda_{n}}(x) \neq \varnothing$. We choose $r^{n}(x) \in R^{n}(x)$ such that $M r^{n}(x) \notin R^{n}(x)$. From the definition of $\eta$ we have $2 M K_{0} \eta=1 / K_{0}-\eta$. We also know that $r^{n}(x) \in R^{n}(x) \subset\left(0,2 K_{0} \eta R_{1}\right]$, then $M r^{n}(x) \leq 2 K_{0} \eta M R_{1}=$ $\left(1 / K_{0}-\eta\right) R_{1}$ and therefore, $\beta\left(x, M r^{n}(x)\right) \leq \lambda_{n}$. So (2.7) is proved for $k=n$. Let us assume that $r^{n}, \ldots, r^{k+1}, k \geq 1$, satisfy (2.7) and (2.8). Let $y \in \Omega^{k}$. If $y \notin \Omega^{k+1}$ then we can get $r^{k}(y)$ in the same way as we have got $r^{n}$. If $y \in \Omega^{k+1}$, set $R=R^{k}(y)-\left[0, r^{k+1}(y)\right)$. Let $r^{k}(y) \in R$ such that $M r^{k}(y) \notin R$. Also $M r^{k}(y) \leq$ $\left(1 / K_{0}-\eta\right) R_{1}$ and $M r^{k}(y)>r^{k+1}(y)$, then $\beta\left(y, M r^{k}(y)\right) \leq \lambda_{k}$.

The preceding lemma and the Covering Lemma (2.1) can be applied together in order to obtain the following result. 
(2.9) LEMMA. Let $\left\{\lambda_{k}\right\}_{k=1}^{n}$ be such that $\lambda_{k+1}>\lambda_{k}>0$. Then for each $k=$ $1,2, \ldots, n$ there exists a sequence $\left\{x_{i}^{k}: i \in \mathbf{Z}^{+}\right\} \subset \Omega^{k}$ such that:

$$
B\left(S\left(x_{i}^{k}, r^{k}\left(x_{i}^{k}\right)\right)\right) \cap B\left(S\left(x_{j}^{k}, r^{k}\left(x_{j}^{k}\right)\right)\right)=\varnothing \quad \text { if } i \neq j ;
$$

(2.11) for every $x \in \Omega^{k}$ there exists $i \in \mathbf{Z}^{+}$such that $\tau\left(x, r^{k}(x)\right) \leq 2 \tau\left(x_{i}^{k}, r^{k}\left(x_{i}^{k}\right)\right)$ and

$$
\begin{gathered}
B\left(S\left(x, r^{k}(x)\right)\right) \subset B\left(\sigma\left(x_{i}^{k}, r^{k}\left(x_{i}^{k}\right)\right) ; 5 K_{0}^{2} \tau\left(x_{i}^{k}, r^{k}\left(x_{i}^{k}\right)\right)\right) ; \\
\beta\left(x_{i}^{k}, r^{k}\left(x_{i}^{k}\right)\right)>\lambda_{k} \geq \beta\left(x_{i}^{k}, M r^{k}\left(x_{i}^{k}\right)\right), \quad \text { for every } i \in \mathbf{Z}^{+} ; \\
\Omega^{k} \subset \bigcup_{i=1}^{\infty} B\left(\sigma\left(x_{i}^{k}, r^{k}\left(x_{i}^{k}\right)\right) ; N \tau\left(x_{i}^{k}, r^{k}\left(x_{i}^{k}\right)\right)\right),
\end{gathered}
$$

where $N$ depends only on $K_{i} i=0,1,3$.

(2.14) Let $j \in \mathbf{Z}^{+}$, then there exists $i \in \mathbf{Z}^{+}$such that

$$
B\left(S\left(x_{j}^{k+1}, r^{k+1}\left(x_{j}^{k+1}\right)\right)\right) \subset B\left(x_{i}^{k}, M r^{k}\left(x_{i}^{k}\right)\right) \subset B\left(\sigma\left(x_{i}^{k}, r^{k}\left(x_{i}^{k}\right)\right) ; P \tau\left(x_{i}^{k}, r^{k}\left(x_{i}^{k}\right)\right)\right),
$$

where $P$ depend only on the $K_{i}$ 's.

PROOF. For each $x \in \Omega^{k}$ we have the positive number $r^{k}(x)$ satisfying (2.7). Applying the lag mapping $S$ to the pair $\left(x, r^{k}(x)\right)$ we get $\left(\sigma\left(x, r^{k}(x)\right) ; \tau\left(x, r^{k}(x)\right)\right)$, which defines the ball $B\left(\sigma\left(x, r^{k}(x)\right) ; \tau\left(x, r^{k}(x)\right)\right)$. In this way we have the family of balls

$$
B^{k}=\left\{B\left(\sigma\left(x, r^{k}(x)\right) ; \tau\left(x, r^{k}(x)\right)\right): x \in \Omega^{k}\right\} .
$$

It is clear that the set defined by the union of all members of $B^{k}$ is bounded. So that we can apply the Covering lemma in order to obtain a sequence $\left\{x_{i}^{k}\right\} \subset \Omega^{k}$ satisfying (2.10), (2.11) and (2.12). Let us prove (2.13): let $x \in \Omega^{k}$ and $i$ be the positive integer associated to $x$ by $(2.11)$, then from (2.3) we have

$$
\begin{aligned}
d\left(x, \sigma\left(x_{i}^{k}, r^{k}\left(x_{i}^{k}\right)\right)\right) & \leq K_{0}\left[d\left(x, \sigma\left(x, r^{k}(x)\right)\right)+d\left(\sigma\left(x, r^{k}(x)\right) ; \sigma\left(x_{i}^{k}, r^{k}\left(x_{i}^{k}\right)\right)\right)\right] \\
& <K_{0}\left[K_{1} r^{k}(x)+5 K_{0}^{2} \tau\left(x_{i}^{k}, r^{k}\left(x_{i}^{k}\right)\right)\right] \\
& \leq K_{0}\left[K_{1} K_{3} \tau\left(x, r^{k}(x)\right)+5 K_{0}^{2} \tau\left(x_{i}^{k}, r^{k}\left(x_{i}^{k}\right)\right)\right] \\
& \leq K_{0}\left[2 K_{1} K_{3}+5 K_{0}^{2}\right] \cdot \tau\left(x_{i}^{k}, r^{k}\left(x_{i}^{k}\right)\right),
\end{aligned}
$$

and (2.13) is proved. In order to prove (2.14) let us observe that, because of (2.11), there exists $i \in \mathbf{Z}^{+}$such that $\tau\left(x_{j}^{k+1}, r^{k}\left(x_{j}^{k+1}\right)\right) \leq 2 \tau\left(x_{i}^{k}, r^{k}\left(x_{i}^{k}\right)\right)$ and

$$
B\left(S\left(x_{j}^{k+1}, r^{k}\left(x_{j}^{k+1}\right)\right)\right) \subset B\left(\sigma\left(x_{i}^{k}, r^{k}\left(x_{i}^{k}\right)\right) ; 5 K_{0}^{2} \tau\left(x_{i}^{k}, r^{k}\left(x_{i}^{k}\right)\right)\right) .
$$

Let $x \in B\left(S\left(x_{j}^{k+1}, r^{k+1}\left(x_{j}^{k+1}\right)\right)\right)$, we can estimate the distance from $x$ to $x_{i}^{k}$ applying the triangle inequality to the following chain of points

$$
x \rightarrow \sigma\left(x_{j}^{k+1}, r^{k+1}\left(x_{j}^{k+1}\right)\right) \rightarrow x_{j}^{k+1} \rightarrow \sigma\left(x_{j}^{k+1}, r^{k}\left(x_{j}^{k+1}\right)\right) \rightarrow \sigma\left(x_{i}^{k}, r^{k}\left(x_{i}^{k}\right)\right) \rightarrow x_{i}^{k},
$$

so that, from (2.3) and (2.8) we have

$$
\begin{aligned}
d\left(x, x_{i}^{k}\right)< & K_{0}^{5}\left[\tau\left(x_{j}^{k+1}, r^{k+1}\left(x_{j}^{k+1}\right)\right)+K_{1} r^{k+1}\left(x_{j}^{k+1}\right)+K_{1} r^{k}\left(x_{j}^{k+1}\right)\right. \\
& \left.\quad+5 K_{0}^{2} \tau\left(x_{i}^{k}, r^{k}\left(x_{i}^{k}\right)\right)+K_{1} r^{k}\left(x_{i}^{k}\right)\right] \\
\leq & K_{0}^{5}\left[\left(K_{2}^{-1}+2 K_{1}\right) r^{k}\left(x_{j}^{k+1}\right)+\left(5 K_{0}^{2} / K_{2}+K_{1}\right) r^{k}\left(x_{i}^{k}\right)\right] \\
\leq & M r^{k}\left(x_{i}^{k}\right) .
\end{aligned}
$$

The last inclusion in (2.14) is easy. 
(2.15) LEMMA. Let $\left\{\lambda_{k}\right\}_{k=0}^{n}$ be such that $\lambda_{k+1}>\lambda_{k}>0$. Then there exists a constant $Q$ depending only on $A$ and the $K_{i}$ 's such that the inequality

$$
\begin{array}{r}
\left(h\left(\lambda_{k+1}\right)-h\left(\lambda_{k}\right)-1\right) \sum_{j=1}^{\infty} \mu\left(B\left(S\left(x_{j}^{k+1}, r^{k+1}\left(x_{j}^{k+1}\right)\right)\right)\right) \\
\leq Q \sum_{i=1}^{\infty} \mu\left(B\left(S\left(x_{i}^{k}, r^{k}\left(x_{i}^{k}\right)\right)\right)\right)
\end{array}
$$

holds for $k=n-1, n-2, \ldots, 1,0$.

PROOF. Set $B_{i}^{* k}=B\left(S\left(x_{i}^{k}, r^{k}\left(x_{i}^{k}\right)\right)\right)$. For every $j \in \mathbf{Z}^{+}$we have $\lambda_{k+1}<$ $\beta\left(x_{j}^{k+1}, r^{k+1}\left(x_{j}^{k+1}\right)\right)$, then, from (2.4) and the properties of $h$ it follows that

$$
h\left(\lambda_{k+1}\right) \leq 1+m_{B_{j}^{* k+1}}(h(f))
$$

consequently

$$
\left(h\left(\lambda_{k+1}\right)-1\right) \sum_{j=1}^{\infty} \mu\left(B_{j}^{* k+1}\right) \leq \sum_{j=1}^{\infty} \int_{B_{j}^{* k+1}} h(f(y)) d \mu(y) .
$$

From (2.14) we know that each $B_{j}^{* k+1}$ is enclosed in some $B\left(x_{i}^{k}, M r^{k}\left(x_{i}^{k}\right)\right)$. Given $i \in \mathbf{Z}^{+}$, set

$$
\begin{aligned}
J_{i}=\left\{j: B_{j}^{* k+1} \subset B\left(x_{i}^{k}, M r^{k}\left(x_{i}^{k}\right)\right) \text { but } B_{j}^{* k+1} \not \subset B\left(x_{l}^{k}, M r^{k}\left(x_{l}^{k}\right)\right)\right. \\
\text { for } l=1,2, \ldots, i-1\} .
\end{aligned}
$$

Then, from (2.10), (2.12), (2.5) and the second inclusion in (2.14) we get

$$
\begin{aligned}
\left(h\left(\lambda_{k+1}\right)-1\right) \sum_{j=1}^{\infty} \mu\left(B_{j}^{* k+1}\right) \leq \sum_{i=1}^{\infty} \sum_{j \in J_{i}} \int_{B_{j}^{* k+1}} h(f) d \mu & \\
& =\sum_{i=1}^{\infty} \int_{\bigcup_{j \in J_{i}} B_{j}^{* k+1}} h\left(f-\beta\left(x_{i}^{k}, M r^{k}\left(x_{i}^{k}\right)\right)+\beta\left(x_{i}^{k}, M r^{k}\left(x_{i}^{k}\right)\right)\right) d \mu \\
& \leq \sum_{i=1}^{\infty}\left\{\int_{B\left(x_{i}^{k}, M r^{k}\left(x_{i}^{k}\right)\right)} h\left(f-\beta\left(x_{i}^{k}, M r^{k}\left(x_{i}^{k}\right)\right)\right) d \mu\right. \\
& \left.+h\left(\lambda_{k}\right) \sum_{j \in J_{i}} \mu\left(B_{j}^{* k+1}\right)\right\} \\
& \leq A \eta^{-\log _{2} A} \sum_{i=1}^{\infty} \mu\left(B\left(x_{i}^{k}, M r^{k}\left(x_{i}^{k}\right)\right)\right)+h\left(\lambda_{k}\right) \sum_{j=1}^{\infty} \mu\left(B_{j}^{* k+1}\right) \\
\leq & Q \sum_{i=1}^{\infty} \mu\left(B_{i}^{* k}\right)+h\left(\lambda_{k}\right) \sum_{j=1}^{\infty} \mu\left(B_{j}^{* k+1}\right) . \quad \square
\end{aligned}
$$

(2.16) LEMMA. There exist positive constants $a$ and $b$ depending only on $A$ and the $K_{i}$ 's such that the inequality

$$
\sum_{i=1}^{\infty} \mu\left(B\left(S\left(x_{i}^{\lambda}, r^{\lambda}\left(x_{i}^{\lambda}\right)\right)\right)\right) \leq a 2^{-b h(\lambda)} \mu\left(B\left(x_{1}, \eta R_{1}\right)\right)
$$

holds for every $\lambda>0$. 
PROOF. Let $Q$ be the constant in Lemma (2.15). Choose $n$ and $r$ such that $h(\lambda)=h(r)+n(2 Q+1)$ and $0 \leq h(r)<2 Q+1$. Let $\lambda_{k}=h^{-1}(k(2 Q+1)+h(r))$, then we can apply Lemma (2.15) to the sequence $\lambda=\lambda_{n}>\lambda_{n-1}>\cdots>\lambda_{0}=r$. Observe that $h\left(\lambda_{k+1}\right)-h\left(\lambda_{k}\right)-1=2 Q$. Then

$$
\sum_{j \in \mathbf{Z}^{+}} \mu\left(B_{j}^{* k+1}\right) \leq \frac{1}{2} \sum_{j \in \mathbf{Z}^{+}} \mu\left(B_{j}^{* k}\right),
$$

by iteration

$$
\begin{aligned}
\sum_{j} \mu\left(B_{j}^{* n}\right) & \leq 2^{-n} \sum_{j} \mu\left(B_{j}^{* 0}\right) \\
& =2^{h(r) /(2 Q+1)} \cdot 2^{-h(\lambda) /(2 Q+1)} \sum_{j} \mu\left(B_{j}^{* 0}\right) .
\end{aligned}
$$

This finishes the proof of the lemma.

(2.17) LEMMA. There exist two positive constants $a$ and $b$ depending only on $A$ and the $K_{i}$ 's such that the inequality

$$
\mu\left\{x \in B\left(x_{1}, \eta R_{1}\right): f^{+}(x)>\lambda\right\} \leq a 2^{-b h(\lambda)} \cdot \mu\left(B\left(x_{1}, \eta R_{1}\right)\right)
$$

holds for every $\lambda>0$.

Proof. Let us show first that $\left\{x \in B\left(x_{1}, \eta R_{1}\right): f^{+}(x)>\lambda\right\} \subset \Omega^{\alpha}$, where $\alpha=h^{-1}(h(\lambda)-1)$ for $h(\lambda)>1$. Let $x \in B\left(x_{1}, \eta R_{1}\right)$ such that $x \notin \Omega^{\alpha}$, then $\beta(x, r) \leq \alpha$ for every $r<2 \eta K_{0} R_{1}$. Observe now that

$$
\frac{1}{\mu(B(x, r))} \int_{B(x, r)} h(f) d \mu \leq \frac{1}{\mu(B(x, r))} \int_{B(x, r)} h(f-\beta(x, r)) d \mu+h(\alpha) \leq h(\lambda),
$$

by differentiation of the integral we get $f^{+}(x) \leq \lambda$. Now, from Lemma (2.16) and (2.13) with $\alpha$ instead of $\lambda_{k}$, we see that

$$
\begin{aligned}
\mu\left\{x \in B\left(x_{1}, \eta R_{1}\right):\right. & \left.f^{+}(x)>\lambda\right\} \leq \mu\left(\Omega^{\alpha}\right) \\
& \leq \sum_{i} \mu\left(B\left(\sigma\left(x_{i}^{\alpha}, r^{\alpha}\left(x_{i}^{\alpha}\right)\right) ; N \tau\left(x_{i}^{\alpha}, r^{\alpha}\left(x_{i}^{\alpha}\right)\right)\right)\right) \\
& \leq C \sum_{i} \mu\left(B^{* \alpha}\right) \leq a 2^{-b h(\lambda)} \cdot \mu\left(B\left(x_{1}, \eta R_{1}\right)\right) .
\end{aligned}
$$

The result of this section is summed up in the following statement.

(2.18) JOHN-NIRENBERG TYPE LEMMA. Let $T$ be a lag mapping on the ball $B\left(x_{1}, R_{1}\right)$ of the space of homogeneous type $(X, d, \mu)$. Then, there exist $\eta\left(K_{i}\right)>0$, $a\left(A, K_{i}\right)>0$ and $b\left(A, K_{i}\right)>0$ such that for every $f \in \operatorname{BMO}\left(x_{1}, R_{1}, T, h\right)$, the inequality

$$
\begin{gathered}
\mu\left\{x \in B\left(x_{1}, \eta R_{1}\right):\left[f(x)-C\left(x_{1}, R_{1}\right)\right]^{+}>\lambda\right\} \\
\leq a e^{-b h(\lambda) / N(f)} \cdot \mu\left(B\left(x_{1}, \eta r_{1}\right)\right)
\end{gathered}
$$

holds for every $\lambda>0$. 


\section{Proof of Theorem (1.7).}

(3.1) LEMMA. Let $T=(\xi, \rho)$ be a one-to-one lag mapping on $B\left(x_{0}, R_{0}\right)$ satisfying (1.8). Then there exist $\eta\left(K_{i}\right)>0$ and $\delta\left(\gamma, K_{i}\right)>0$ such that $\mathrm{BMO}\left(x_{0}, R_{0}, T, h\right)$ $\subset \mathrm{BMO}\left(x_{0}, \eta \delta R_{0}, T_{\eta}, l\right)$, where $T_{\eta}(x, r)=(\xi(x, r / \eta) ; \eta \rho(x, r / \eta))$ and $l(s)=s^{+}$.

PROOF. Let $f \in \operatorname{BMO}\left(x_{0}, R_{0}, T, h\right)$. If $x \in B\left(x_{0}, R_{0} / 2 K_{0}\right)$ and $r \leq R_{0} / 2 K_{0}$, then $B(x, r) \subset B\left(x_{0}, R_{0}\right)$. So we can apply Lemma (2.18) in order to obtain

$$
\mu\left\{y \in B(x, \eta r):[f(y)-C(x, r)]^{+}>\lambda\right\} \leq a e^{-b h(\lambda) / N(f)} \cdot \mu(B(x, \eta r)),
$$

then, from the integrability hypothesis on $e^{-\varepsilon h}$ we have

$$
m_{B(x, \eta r)}[f-C(x, r)]^{+} \leq N^{\prime}(f)
$$

for every $x \in B\left(x_{0}, R_{0} / 2 K_{0}\right)$ and $r<R_{0} / 2 K_{0}$. Let us now observe that, because of (1.8), $T^{-1}$ is a lag mapping on the ball $B\left(x_{0}, \gamma R_{0}\right)$, and that $-f \in$ $\operatorname{BMO}\left(x_{0}, \gamma R_{0}, T^{-1}, h\right)$ with constants $\tilde{c}(\xi, \rho)=-C\left(T^{-1}(\xi, \rho)\right)=-C(x, r)$. Applying again Lemma (2.18) we get

$$
m_{B(\xi, \eta \rho)}[C(x, r)-f]^{+} \leq N^{\prime}(f),
$$

for every $\xi \in B\left(x_{0}, \gamma R_{0} / 2 K_{0}\right)$ and $\rho<\gamma R_{0} / 2 K_{0}$. If we take $\delta<\gamma K_{2} / 2 K_{0}^{2}\left[K_{1}+1\right]$, then the image under $T$ of $B\left(x_{0}, \delta R_{0}\right) \times\left(0, \delta R_{0}\right)$ is contained in the set for which (3.3) holds. Set $s=\eta r$ in (3.2) and (3.3), we have

$$
m_{B(x, s)}\left[(f-C(x, s / \eta))^{+}\right] \leq N^{\prime}(f)
$$

and

$$
m_{B(\xi(x, s / \eta) ; \eta \rho(x, s / \eta))}\left[(C(x, s / \eta)-f)^{+}\right] \leq N^{\prime}(f),
$$

for every $(x, s) \in B\left(x_{0}, \eta \delta R_{0}\right) \times\left(0, \eta \delta R_{0}\right]$. This finishes the proof of the lemma.

Applying Lemmas (3.1) and (2.18) we obtain the following result.

(3.4) LEMMA. Let $T=(\xi, \rho)$ be a one-to-one lag mapping on $B\left(x_{0}, R_{0}\right)$ satisfying (1.8). Then, there exist $\eta\left(K_{i}\right)>0, \delta\left(\gamma, K_{i}\right)>0, a\left(A, K_{i}\right)>0$ and $b\left(A, K_{i}\right)>0$ such that given $f \in \mathrm{BMO}\left(x_{0}, R_{0}, T, h\right)$ the inequalities

$$
\begin{aligned}
& \mu\left\{y \in B(x, \eta t):[f(y)-C(x, t)]^{+}>\lambda\right\} \leq a e^{-b \lambda / N(f)} \mu(B(x, \eta t)), \\
& \mu\left\{y \in B(\sigma, \eta s):[C(x, t)-f(y)]^{+}>\lambda\right\} \leq a e^{-b \lambda / N(f)} \mu(B(\sigma, \eta s)),
\end{aligned}
$$

hold for every $\lambda>0, x \in B\left(x_{0}, \delta R_{0}\right), t<\delta R_{0}$ and some function $c(x, t) \cdot((\sigma, s)=$ $\left.T_{\eta}(x, t)\right)$.

With these results, the proof of the theorem is now as in the euclidean case: let $\varepsilon<b / N(f), x \in B\left(x_{0}, \delta R_{0}\right)$ and $t<\delta R_{0}$, then

$$
\begin{aligned}
\int_{B(\sigma, \eta s)} u^{\varepsilon} d \mu & \leq e^{-\varepsilon C(x, t)} \int_{B(\sigma, \eta s)} e^{\varepsilon(C(x, t)-f(y))^{+} d \mu(y)} \\
& \leq a e^{-\varepsilon C(x, t)} \varepsilon \int_{0}^{\infty} e^{[\varepsilon-b / N(f)] \lambda} d \lambda \mu(B(\sigma, \eta s)) .
\end{aligned}
$$

On the other hand

$$
\int_{B(x, \eta t)} u^{-\varepsilon} d \mu \leq a e^{\varepsilon C(x, t)} \varepsilon \int_{0}^{\infty} e^{[\varepsilon-b / N(f)] \lambda} d \lambda \mu(B(x, \eta t)) .
$$


Consequently

$$
\left(\int_{B(x, r)} u^{-\varepsilon} d \mu\right)\left(\int_{B(\varsigma, \tau)} u^{\varepsilon} d \mu\right) \leq c \mu(B(x, r))^{2},
$$

for every $r<\eta \delta R_{0}$ and $x \in B\left(x_{0}, \eta \delta R_{0}\right)$, where

$$
(\varsigma, \tau)=S(x, r)=\left(\xi\left(x, r / \eta^{2}\right) ; \eta^{2} \rho\left(x, r / \eta^{2}\right)\right) .
$$

A KNOWLEDGment. The author wishes to express his gratitude to E. B. Fabes and C. Gutierrez for many discussions and helpful comments on the subject.

\section{REFERENCES}

[B] E. Bombieri, Theory of minimal surfaces and a counter-example to the Bernstein conjecture in high dimensions, Lecture Notes, Courant Institute, New York Univ., 1970.

[Bu] N. Burguer, Espace des fonctions a variation moyenne bornee sur un espace de nature homogene, C. R. Acad. Sci. Paris Ser. A 286 (1978), 139-142.

[CS 1 F. Chiarenza and R. Serapioni, A Harnack inequality for degenerate parabolic equations, Comm. Partial Differential Equations 9 (1984), 719-749.

$\left[\mathbf{C S}_{2}\right] \ldots$, Degenerate parabolic equations and Harnack inequality, Ann. Mat. Pura Appl. 87 (1984), 139-162.

[CW] R. Coifman and G. Weiss, Analyse harmonique non-commutative sur certains espaces homogenes, Lecture Notes in Math., Vol. 242, Springer-Verlag, Berlin and New York, 1972.

[FG] E. Fabes and N. Garofalo, Parabolic B.M.O. and Harnack's inequality, Proc. Amer. Math. Soc. 95 (1985), 63-69.

[FKS] E. Fabes, C. Kenig and R. Serapioni, The local regularity of solutions of degenerate elliptic equations, Comm. Partial Differential Equations 7 (1982).

[FL] B. Franchi and E. Lanconelli; Holder regularity theorem for a class of linear non uniformly elliptic operators with measurable coefficients Ann. Scuola Norm. Sup. Pisa (4) 10 (1983), 523-541.

[FS] B. Franchi and R. Serapioni, Pointwise estimates for a class of strongly degenerate elliptic operators: a geometrical approach, Università Degli Studi di Trento, Italy, U.T.M. 195, 1986, pp. $1-57$.

[JN] F. John and L. Nirenberg, On functions of bounded mean oscillation, Comm. Pure Appl. Math. 14 (1961), 415-426.

[M1] J. Moser, On Harnack's theorem for elliptic differential equations, Comm. Pure Appl. Math. 14 (1961), 577-591.

[M2] _ A Harnack inequality for parabolic differential equations, Comm. Pure Appl. Math. 17 (1964), 101-134; Correction to "A Harnack inequality for parabolic differential equations", Comm. Pure Appl. Math. 20 (1967), 232-236.

[M3] _ On a pointwise estimate for parabolic differential equations, Comm. Pure Appl. Math. 24 (1971), 727-740.

[MS] R. Macias and C. Segovia, A well-behaved quasi-distance for spaces of homogeneous type, Trabajos de Matematica, Vol. 32, Inst. Argentino Mat., 1981, pp. 1-18.

[MW] B. Muckenhoupt and R. Wheeden, Weighted bounded mean oscillation and the Hilbert transform, Studia Math. 54 (1976), 221-237.

[N] U. Neri, Some properties of functions with bounded mean oscillation, Studia Math. 61 (1977), 63-75.

Department of Mathematics, University of Minnesota, Minneapolis, MinNESOTA 55455

Programa Especial de Matemática Aplicada, Santa fe, Argentina

Current address: INTEC, Guemes 3450, 3000 Santa Fe, Argentina 